\title{
Biologically Enabled Micro- and Nanostencil Lithography Using Diatoms
}

Jun Cai ${ }^{1}$, Xiaoning Wang ${ }^{1}$, Aobo Li ${ }^{1}$, Stephan W. Anderson ${ }^{2}$, and Xin Zhang ${ }^{1}$

1. Department of Mechanical Engineering, Boston University, Boston, MA (USA)

2. Department of Radiology, Boston University Medical Center, Boston, MA (USA)

\section{Corresponding Authors}

S.W. Anderson, MD

820 Harrison Avenue, Boston, MA 02118, USA

Phone: +1(617)638-6610

E-mail: Stephan.Anderson@bmc.org

X. Zhang, PhD

110 Cummington Mall, Boston, MA 02215, USA

Phone: +1(617)358-2702

E-mail: xinz@bu.edu

\section{Present Addresses}

J. Cai, PhD

School of Mechanical Engineering \& Automation

Beihang University, Beijing, China

\section{ABSTRACT}

The development of a biologically enabled micro- and nanostencil lithography approach using diatoms is demonstrated. Diatom frustules are initially purified, sorted, and aligned into compact monolayers on underlying silicon substrates. Subsequently, the diatom monolayers are employed as shadow masks during the electron beam deposition of gold ( $\mathrm{Au}$ ) thin films, a process which enables the capacity to mirror the intricate micro- and nanoporous frustule architecture on the underlying silicon substrates. Following Au deposition and diatom frustule dissolution, both submicron- and nanoscale gold patterns on silicon are realized using this approach. This unique method yields the highly structured patterning of gold and other materials on a variety of substrates, with feature sizes ranging from the sub-micron to the nanoscale, enabling a host of diverse applications.

KEYWORDS: diatoms, nanofabrication, microfabrication 


\section{INTRODUCTION}

Diatoms are microscopic, unicellular photosynthetic algae which are ubiquitous in aquatic environments, with approximately 100,000 different species ranging from $1 \mu \mathrm{m}$ to $4 \mathrm{~mm}$ in size (1). The silica exoskeletons of diatoms, termed frustules, not only have thousands of distinct morphologies but also possess a wide range of intricate micro- and nanoscale patterns. The unique morphologies of these biologically evolved exoskeletons are optimized for a variety of functions including mechanical protection, gas and nutrient exchange, as well as offering mechanical protection $(2,3)$. Given their highly organized morphology, diatoms represent promising candidates for developing biologically assisted fabrication approaches. A further consideration regarding the potential of these organisms in nanofabrication is the capacity to exert a degree of control over the morphology of the diatom exoskeleton using genetic engineering approaches. Since the initial sequencing of the diatom genome, the door towards rationally designing three-dimensional nanostructures by manipulating the diatom genome has been opened and offers a tremendous potential for biologically-enabled technology (4, 5). To date, the capacity to replicate the diatom morphology using various molding and templating approaches has been reported, thereby establishing the potential of these biological templates to enable novel micro- and nanofabrication strategies $(6,7)$. In these instances, the nanoporous morphology of the diatom frustules has been employed to yield nanotextured substrates composed of metal as well as polydimethylsiloxane (PDMS) (8-10). Beyond the capacity to generate nanotextured surfaces, however, the use diatoms to create micro- and nanoscale patterns of various materials, such as metals or polymers, on a variety of underlying substrates has not been demonstrated. This capacity to create patterns of materials which mirror the intricate morphology of these biological templates has the potential to facilitate a variety of practical 
applications and will be specifically addressed herein (11). Furthermore, the majority of the prior efforts which employed diatoms in biologically enabled fabrication strategies have focused on either collections of randomly distributed diatoms or single diatoms (8-10). However, with the exception of a previously reported method using inkjet printing of diatom frustules, there has been little progress in the development of fabrication strategies which employ uniformly arrayed diatom frustules, a critical step towards the realization of scalable diatom-based micro/nanomanufacturing and another important focus of this work (12). In this letter, we report the development of a versatile, diatom-enabled fabrication approach with the capacity to generate precisely patterned gold micro- and nanostructures which mirror the highly-organized, porous frustule morphology on underlying silicon substrates. Specifically, Coscinodiscus $s p$. diatom frustules were uniformly aligned on silicon substrates and employed as shadow masks in a stencil lithography approach during the electron beam deposition of gold (Au). This novel, biologically-enabled stencil lithography approach yielded the capacity to precisely pattern gold structures on the silicon substrate over large areas with a range of feature sizes, ranging down to the nanoscale.

\section{EXPERIMENTAL}

Purification and separation of diatom frustule: The diatom Coscinodiscus sp. (CCMP 1583) was purchased from the National Center for Marine Algae and Microbiota (NCMA, formerly CCMP) at the Bigelow Laboratory for Ocean Sciences. The Coscinodiscus sp. diatoms were harvested from $2 \mathrm{~L}$ of culture media using a sieve, yielding approximately of $10 \mathrm{~mL}$ of concentrated diatoms. The concentrated diatom solution was gradually poured into a beaker containing 100 $\mathrm{mL}$ of concentrated sulfuric acid $\left(98 \% \mathrm{H}_{2} \mathrm{SO}_{4}\right)$-based pickling solution. Subsequently, this solution was heated in a water bath at $65^{\circ} \mathrm{C}$ for 40 min to ensure the complete removal of all the 
organic contents of diatoms. After the solution was cooled, the diatom exoskeletons were filtered with a cell strainer (40 $\mu \mathrm{m}$ in mesh size, 352340, BD Falcon) and subsequently continuously flushed with deionized water for 5 minutes. The cleaned frustule valves and girdle bands were then again dispersed in deionized water and filtered with another cell strainer $(70 \mu \mathrm{m}$ in mesh size, 352350, BD Falcon) to remove any frustule components exceeding $70 \mu \mathrm{m}$ in size. Next, a series of settling processes were used to separate the diatom valves from the girdle bands (11). In order to eliminate nanopore layer along the outer surface of the diatom valves, the diatom frustules were further treated with $0.1 \% \mathrm{HF}$ solution for $1 \mathrm{~min}$, then filtered with a cell strainer $(40 \mu \mathrm{m})$ and flushed thoroughly with deionized water.

Assembly and lithography of diatom frustules mono layer: A $20 \mathrm{~mm}$ x $20 \mathrm{~mm}$ silicon wafer was immersed in $10 \%$ HF solution for 10 min to eliminate the silica film, after which it was flushed with deionized water for $1 \mathrm{~min}$ and then blown dry with compressed air flow. Approximately $500 \mu \mathrm{l}$ of a condensed diatom frustule solution ( 2000 frustules $/ \mathrm{ml})$ was pipetted onto the asprepared silicon wafer to form a small pool, after which the wafer was placed on a heating plate at $130^{\circ} \mathrm{C}$ to accelerate the water evaporation. The solution was heated to the water boiling point in approximately $1 \mathrm{~min}$, yielding the continuous generation of bubbles along the substrate surface.

Next, thin gold films (10 nm to $20 \mathrm{~nm}$ in thickness) were deposited at a rate of $0.05 \mathrm{~nm} / \mathrm{s}$ in an electron beam evaporation deposition machine (Solution, CHA Industries, CA). Subsequently, the diatoms were completely removed by etching using a $1 \%$ HF solution for 5 min. After HF etching and flushing the substrate with deionized water, only the patterned gold array persisted on the silicon substrate. Figure 1 demonstrates the process flow during which gold thin films 
were patterned upon the diatom monolayers, followed by dissolution of the diatoms, yielding a replication of the intricate porous pattern of the diatom valves.

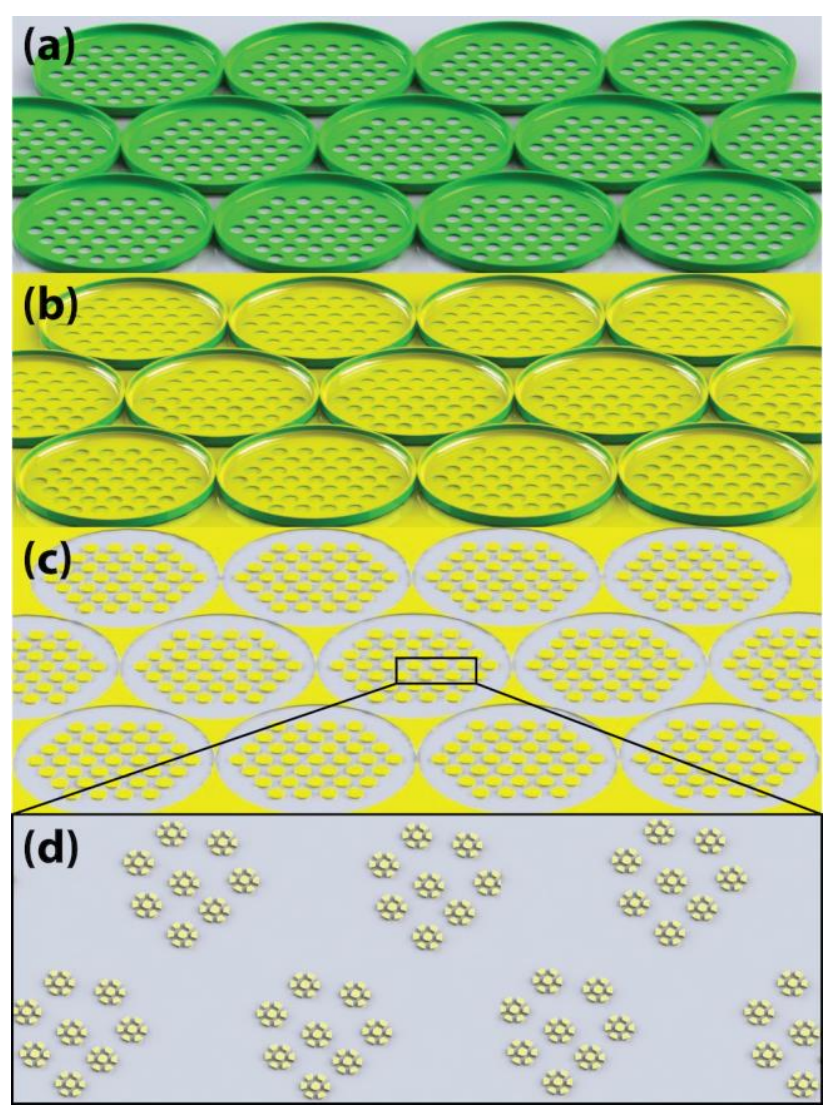

Figure 1. Illustration of diatom assembly and stencil lithography approach. (a) Assembled monolayer of diatom frustules on the silicon wafer; (b) Deposit gold layer with electron beam evaporation; (c) Removal of diatom frustules with HF solution, yielding deposited gold layer; (d) Magnification of Fig. 2(c) demonstrating a detailed view of the final gold pattern.

Characterization of diatom frustules and patterned gold arrays: A thin gold-platinum layer (approximately $5 \mathrm{~nm}$ in thickness) was deposited on dried frustules on the silicon wafers, after which SEM (Supra 55VP FESEM from Carl Zeiss Microscopy, NY, USA) was used to investigate the morphology of diatom frustules. The patterned gold arrays on the silicon wafers following diatom dissolution were directly observed with SEM. 


\section{RESULTS}

Of fundamental importance to the approach developed herein are the initial diatom sorting steps which yield highly monodisperse diatom valves for subsequent alignment upon an underlying substrate. Herein, following an initial cleaning step, frustules were double- filtered to ensure a relatively monodisperse size distribution, followed by a series of settling processes to separate the two major components of the diatom frustule, the girdle bands and the diatom valves (13). Figure $2 \mathrm{a}$ and $\mathrm{b}$ demonstrate the cleaned diatom frustules before and after filtration and separation. The detailed morphology of the unique multi-layered porous structure of the diatom valves is shown in Figure 2c-g; a diagram of a cross-section of the diatom frustule (Figure $2 \mathrm{~h}$ ) illustrates its hierarchical structure. 


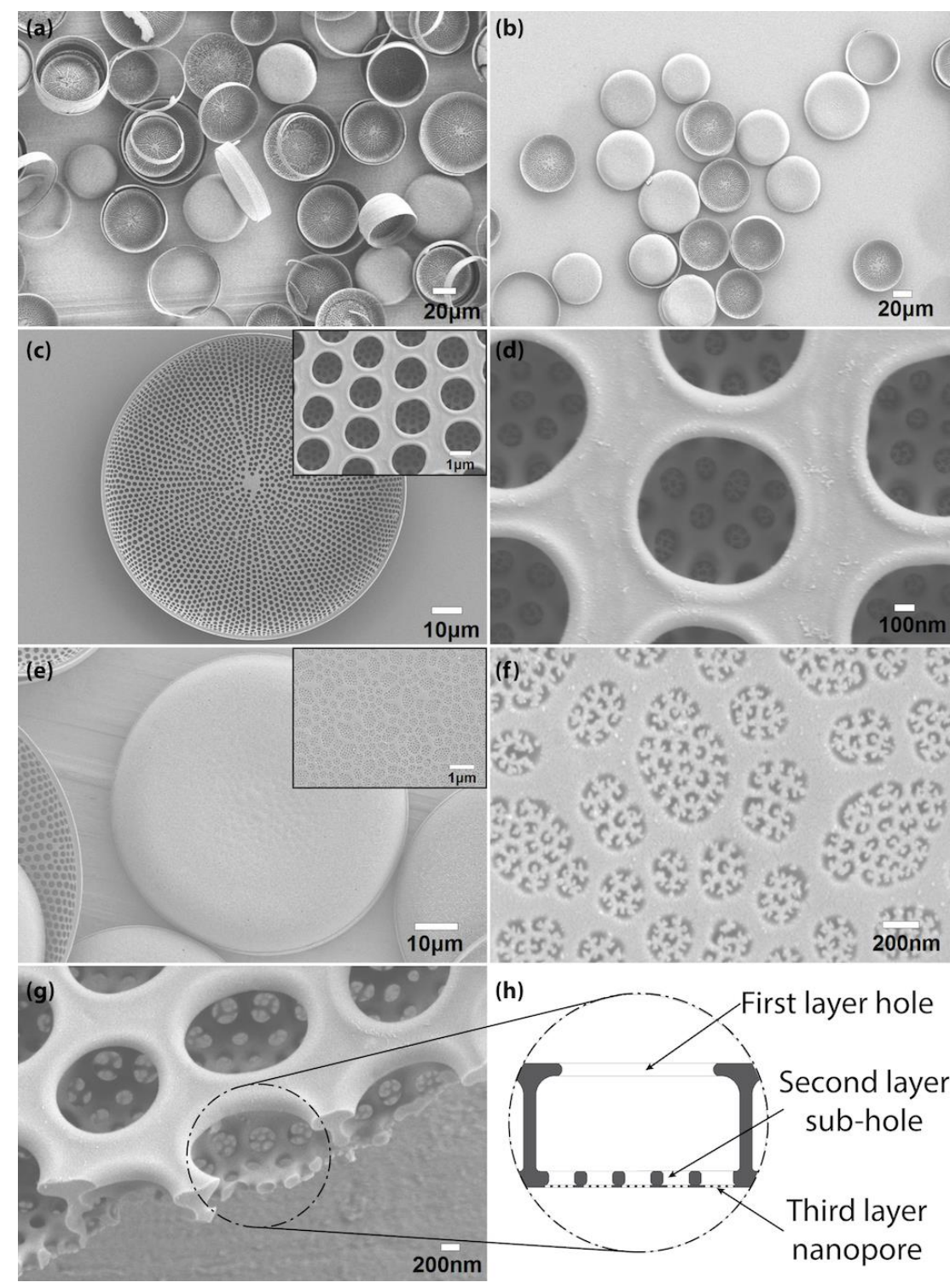

Figure 2. SEM images of diatom frustule morphology. (a) Mixture of diatom frustules and girdle bands obtained after pickling and purification; (b) Randomly distributed diatom frustule valves after separating and screening; (c, d) Overview and detailed view of morphology of single frustule with concave side upwards, insert represents magnified view; (e, f) Overview and detailed view of morphology of single frustule with convex side upwards, insert represents magnified view; (g) Detailed structure of diatom frustules. (h) Diagram demonstrating a crosssection of the frustule valve with hierarchical, three-layered structure.

Following the isolation of high-purity, cleaned diatom valves, some of the diatom frustules were treated with hydrofluoric acid in order to tune the size of the nanopores. In this step, we 
successfully removed the first nanopore layer of the frustules. Figure 3 demonstrates the diatom pores before (Figure $3 \mathrm{a}$ and $\mathrm{b}$ ) and after (Figure $3 \mathrm{c}$ and $\mathrm{d}$ ) the acid cleaning. With the method we are able to exploit frustules' flexibility in stencil lithography by controlling the size of the nanopores of diatoms.
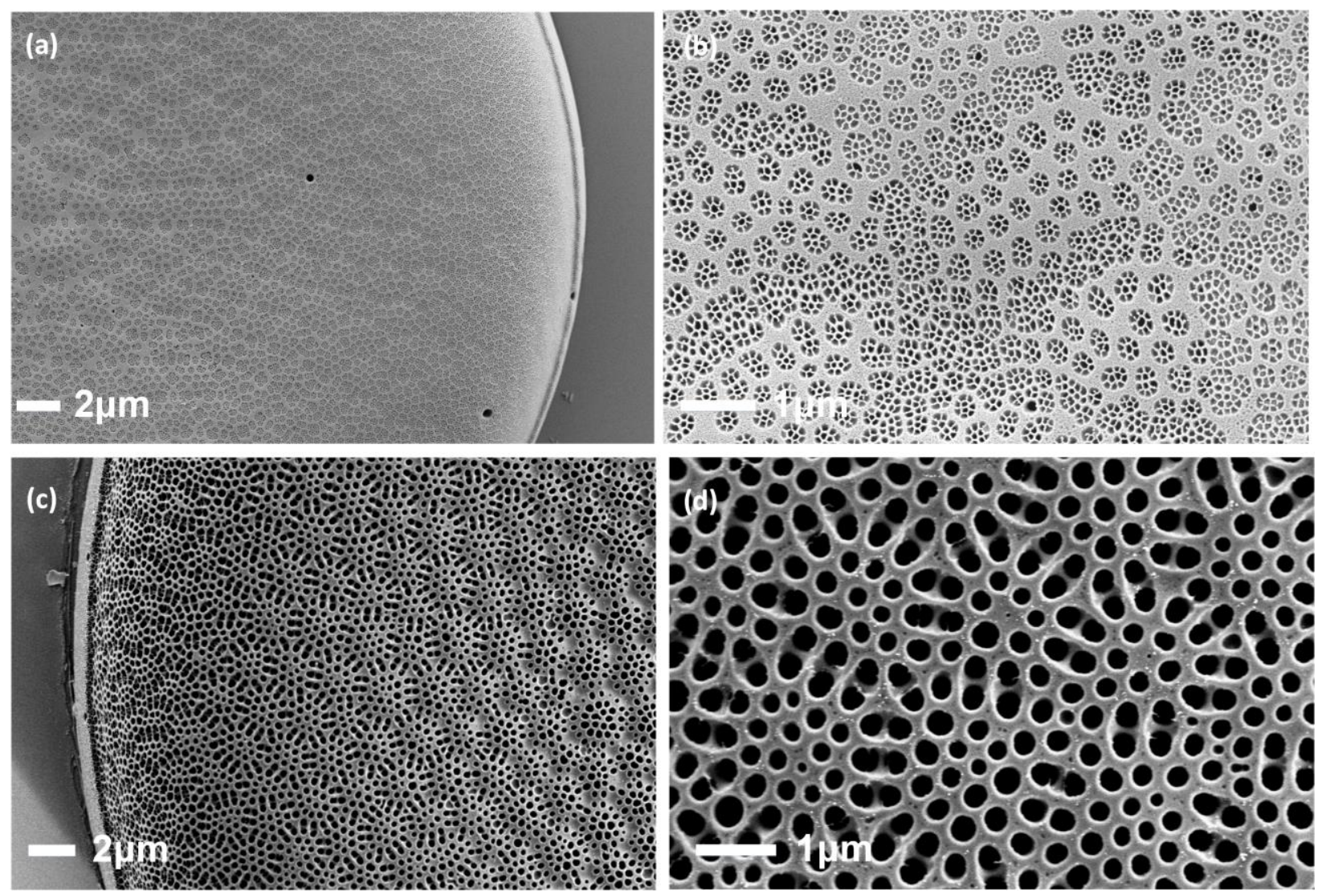

Figure 3. Diatom nanostructures before and after hydrofluoric acid cleaning. (a) Surface nanostructures of a diatom frustule; (b) Zoomed view of the intact nanostructures; (c) Removal of diatom frustules' layer of nanostructures with HF solution, yielding nanopores with larger diameters; (d) Zoomed view of the diatom pore structures after acid cleaning.

An assembly process was then employed to yield large-area, close-packed diatom monolayers. Herein, using a simplified version of the 'floating assembly' approach which we have previously described, the capacity for diatom valves to self-assemble was leveraged (14). Specifically, aqueous solutions bearing high-concentrations of purified diatom valves were pipetted onto silicon wafers and, following quick evaporation of the water by heating the sample to the water 
boiling point, the diatom valves were found to self-assemble in a predominately 'concave-up' orientation in which the concave surfaces of the diatom valves were found to face upward with respect to the underlying substrate. Using this approach, we readily achieved 'concave-up' monolayers of diatoms of up to approximately $4 \mathrm{~mm}^{2}$. Figure 4 shows the comparison between the monolayers from the quick evaporation method and the control group (slowly dried). The figures show that in the experimental group the frustules were arranged with their concave side up, forming a compact monolayer on substrate surface. While in the other case, the diatoms on the substrate were randomly arranged. Overlap between diatoms can be observed, and the diatoms did not form a compact layer.
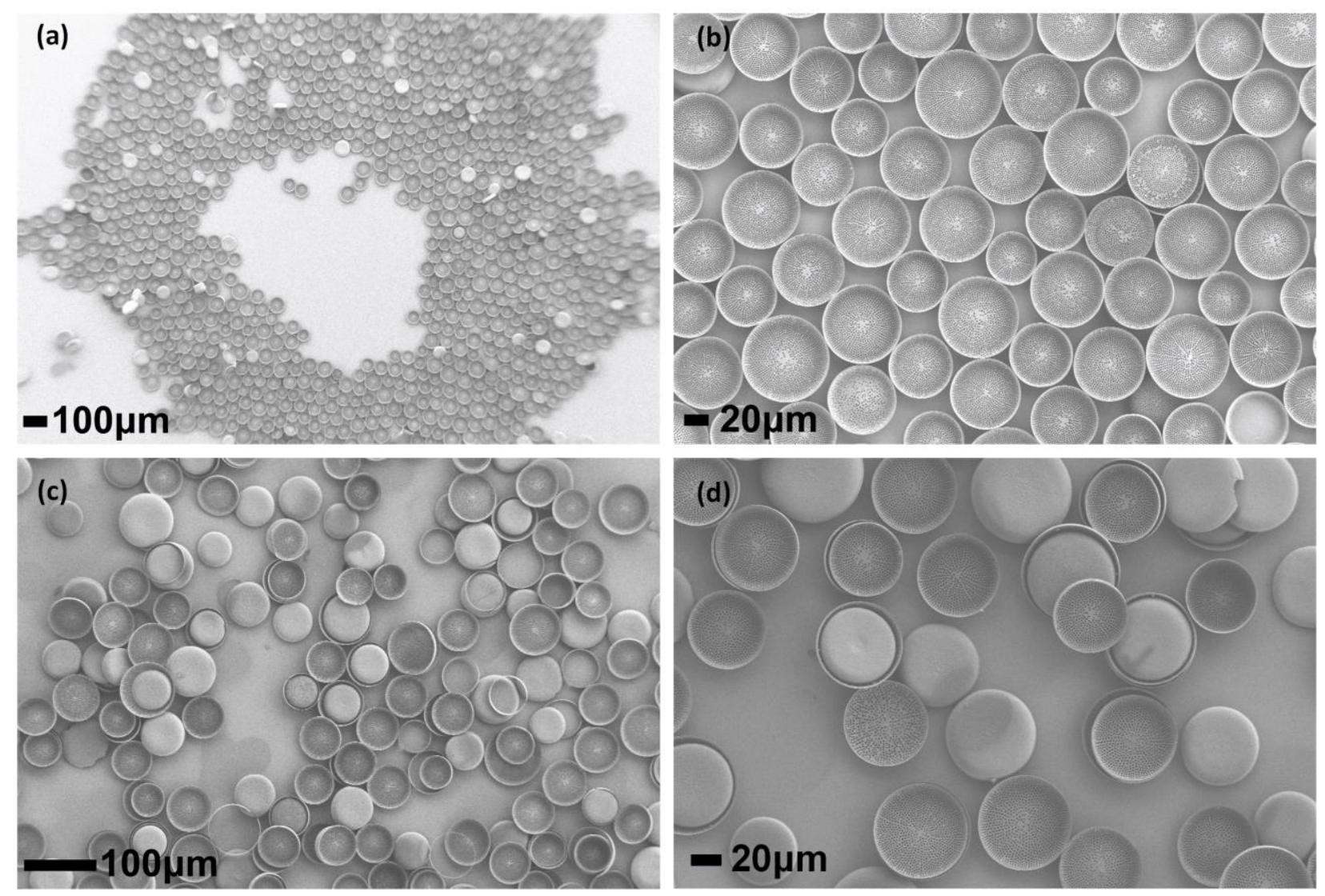

Figure 4. Diatom manipulation with and without fast evaporation method. (a) Compact "concave-up" diatom frustule monolayer after fast evaporation arranging; (b) Zoomed view of the concave-up diatom frustules; (c) Discrete and overlapping diatom frustules dried without fast evaporation; (d) Zoomed view of the randomly distributed diatom frustules. 
These diatom monolayers were subsequently employed as shadow masks in a stencil lithography process during which gold thin films were deposited using electron beam evaporation. Figure 5 demonstrates the appearance of the substrate following diatom dissolution. In this case, the gold nanopatterns demonstrate feature sizes on the order of $20-40 \mathrm{~nm}$, mirroring both the size and pattern of the nanopores of the diatom frustule valves (Figure $5 \mathrm{a}$ and $\mathrm{b}$ ). Of note, with the deposition of gold thin films with thicknesses of $10-20 \mathrm{~nm}$, as employed in this work, distinct nano-scale features reflecting the sizes of the nanopores were seen.

In addition to the gold nanopatterns, the modified diatom valves were able to yield patterns with larger, sub-micron feature sizes. To this end, the high-purity, cleaned diatom valves were subjected to a brief treatment using hydrofluoric acid in order to etch the outermost portions of the valves, resulting in a complete removal of the nanopore layer. In this case, following valve assembly and gold deposition, patterns with feature sizes on the order of $200 \mathrm{~nm}$ were achieved (Figure 5c and d), reflecting both the size and periodicity of the second layer of the diatom valve holes. 

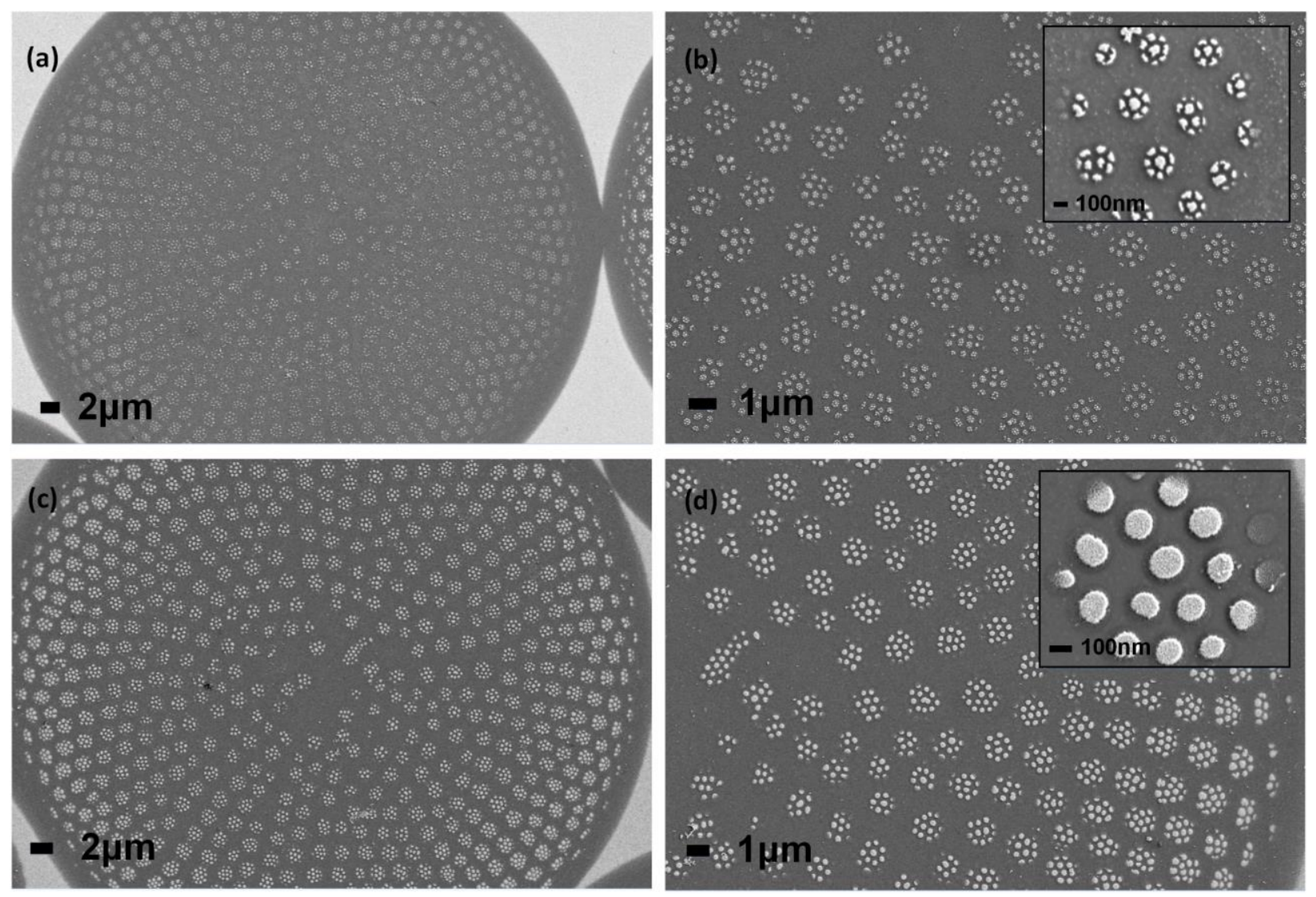

Figure 5. SEM of gold micro- and nanostructures patterned using diatom enabled stencil lithography approach. (a) Overview of patterned gold nanostructures; (b) Zoomed view of patterned gold nanostructures, inserted picture is a magnified view of nanoscale feature sizes; (c) Overview of patterned gold sub-micron structures fabricated after initial elimination of diatom nanopores; (d) Zoomed view of the sub-micron structures, inserted picture is a magnified view of micron-scale feature sizes.

\section{DISCUSSION}

With the fast evaporation method, large area "concave up" diatom frustule monolayer were achieved. While the principle of the formation of the monolayers remain unclear, it is hypothesized that these bubbles resulted in a perturbation of the frustules, causing them to orient in more stable 'concave-up' orientation along the substrate. This hypothesis is supported by the fact that condensed diatom frustule solutions pipetted onto silicon and allowed to dry at room temperature resulted in assemblies in which 'concave-up' and 'concave-down' orientations were represented nearly equally. Furthermore, during the evaporation of the water, the surface tension 
of the droplets was found to drive the diatom frustules to form a compact monolayer on the silicon wafers.

Gold nanopatterns were successfully fabricated from the diatom frustules and the ability to tune the size of the gold patterns was demonstrated. However, we hypothesize that with increasing thin film thickness, the capacity to fabricate distinct replicas of the nanopores of the frustule valves may be compromised as the smallest feature sizes merge into a reflection of the larger, sub-micron feature sizes of the second pore layer.

The diatom enabled stencil lithography approach reported herein which was specifically employed to fabricate precisely patterned gold structures reflecting the micro- and nanoscale features of the diatom frustule may find application to a range of photonics applications such as surface enhanced Raman scattering (SERS). In fact, based on both theoretical and experimental investigation, it was recently demonstrated that the uniquely structured silica exoskeletons of the diatoms offer tremendous potential for fabricating SERS substrates $(15,16)$. Thus, the diatom enabled stencil lithography approach reported herein, given its capacity to pattern a landscape of rationally designed gold micro- and nanostructured features has the potential to be uniquely suited towards the scalable fabrication of SERS substrates. Beyond photonics applications, the capability to fabricate a precisely patterned landscape of micro- and nanoscale features of various materials using a diatom enabled stencil lithography technique potentially enables a wide array of applications, including engineered surface patterning for reducing friction for MEMS/NEMS devices, precisely tailoring various surface for achieving extremely hydrophobic or hydrophilic properties, photovoltaic devices, and a host of biomedical technologies such as biosensing using functionalized gold nanopatterned substrates, among others (17-20). 
Finally, it is instructive to consider the potential advantages of the biologically enabled approach reported herein in comparison to alternative approaches to large area nanopatterning. While enabling the requisite precision to create nanopatterned surfaces, conventional nanolithography techniques such as electron-beam lithography or focused ion beam approaches are fundamentally serial in nature and lack the scalability required for many practical applications. Beyond conventional nanolithography approaches, a host of nanoimprint lithography-based techniques have been developed for diverse applications, including step and flash imprint lithography (SFIL), among others $(21,22)$. However, while imprint lithography approaches yield the requisite precision and their parallel, scalable nature achieves the capacity for increasingly large-area nanopatterning, there remains an inherent need for master molds which are typically fabricated using costly, serial nanolithographic approaches such as electron-beam lithography.

As an alternative to these 'top-down' approaches to nanopatterning, a host of 'bottom-up', selfassembly-based methods have been developed. These 'bottom-up' methods, including such techniques as block copolymer self-assembly and DNA-based nanopatterning, among others, offer a highly scalable approach to nanomanufacturing but lack a similar degree of control over and variety of the patterned nanoscale features when compared to the aforementioned 'top-down' approaches $(23,24)$.

In comparison to the diverse array of alternative approaches noted above, the diatom enabled stencil lithography approach reported herein mitigates several existing limitations of these techniques. The proposed diatom-based approach is envisioned to be yield the requisite scalability to enable a diverse array of applications given the capacity to assemble these naturally occurring templates into large-area arrays. Of note, while the diatom frustule assembly approach reported herein enables the assembly of monolayers of diatoms and, therefore, patterned gold 
arrays, on the order of several millimeters in size, the development of techniques which yield larger assembly of monolayers of diatoms will serve to markedly potentiate this diatom-enabled lithography approach and is an area which deserves ongoing inquiry. Towards this end, the capacity for diatom self-assembly demonstrated herein offers a potential avenue towards the further development of techniques which yield diatom monolayers of increasing size.

In addition to the scalability of diatom enabled lithography given its biological templates, leveraging the staggering variety of nanoscale features which exist in these naturally occurring organisms yields a large degree of freedom in the achievable nanopatterns. Furthermore, as reported herein, the diatom frustules may be modulated prior to monolayer assembly using HF to widen or completely eliminate layers of pores. Alternatively, deposition methods (such as atomic layer deposition, ALD) may be employed to narrow the pore dimensions, yielding additional degrees of freedom during subsequent diatom enabled stencil lithography.

\section{OUTLOOK}

This paper reports a novel micro- and nanostencil lithography approach using diatom frustules which enables the capacity to precisely pattern gold over large areas with feature sizes ranging from the sub-micron to nanoscale. Beyond the materials employed herein, this biologically enabled approach may be readily tailored to pattern a variety of materials on various substrates and has the potential to represent a next generation micro- and nanomanufacturing technology which may be optimized towards a diverse array of applications.

\section{ACKNOWLEDGEMENTS}

The authors would like to thank the National Cancer Institute (Grant No. R25 CAI53955), the National Science Foundation (Grant No. ECCS 1202304), the Center for Nanoscience and Nanobiotechnology at Boston University, and the Photonics Center at Boston University for the 
funding and technical support. J. Cai would like to acknowledge the fellowship support from China Scholarship Council (CSC).

\section{REFERENCES}

1. Parkinson, J.; Gordon, R. Beyond micromachining: the potential of diatoms. Trends Biotechnol. 1999, 17, 190-196.

2. Bradbury, J. Nature's nanotechnologists: unveiling the secrets of diatoms. PLoS Biol. 2004, 2, e306.

3. Hamm, C. E.; Merkel, R.; Springer, O.; Jurkojc, P.; Maier, C.; Prechtel, K.; Smetacek, V. Architecture and material properties of diatom shells provide effective mechanical protection. Nature, 2003, 421, 841-843.

4. Armbrust, E. V.; Berges, J. A.; Bowler, C.; Green, B. R.; Martinez, D. Putnam, N. H.;

Zhou. S.; Allen, A. E.; Apt, K. E.; Bechner, M.; Brzezinski, M. A.; Chaal, B. K.; Chiovitti, A.;

Davis, A. K.; Demarest, M. S.; Detter, J. C.; Glavina, T.; Goodstein, D.; Hadi, M. Z.; Hellsten, U.; Hildebrand, M.; Jenkins, B. D.; Jurka, J.; Kapitonov, V. V.; Kröger, N.; Lau, W. W.; Lane, T. W.; Larimer, F. W.; Lippmeier, J. C.; Lucas, S.; Medina, M.; Montsant, A.; Obornik, M.; Parker, M. S.; Palenik, B.; Pazour, G. J.; Richardson, P. M.; Rynearson, T. A.; Saito, M. A.; Schwartz, D. C.; Thamatrakoln, K.; Valentin, K.; Vardi, A.; Wilkerson, F. P.; Rokhsar, D. S. The genome of the diatom Thalassiosira pseudonana: ecology, evolution, and metabolism. Science, 2004, 306, 79-86.

5. Kröger, N. Prescribing diatom morphology: toward genetic engineering of biological nanomaterials. Current opinion in chemical biology, 2007, 11, 662-669.

6. Sumper, M.; Brunner, E. Learning from Diatoms: Nature's Tools for the Production of Nanostructured Silica. Adv. Funct. Mater. 2006, 16, 17-26.

7. Bozarth, A.; Maier, U. G.; Zauner, S. Diatoms in biotechnology: modern tools and applications. Appl. Microbiol. Biotechnol. 2009, 82, 195-201.

8. Losic, D.; Mitchell, J. G.; Voelcker, N. H. Complex gold nanostructures derived by templating from diatom frustules. Chem. Commun. 2005, 39, 4905-4907.

9. Losic, D.; Mitchell, J. G.; Lal, R.; Voelcker, N. H. Rapid prototyping of micro- and nanoscale patterns by replica moulding from diatom biosilica. Adv. Funct. Mater. 2007, 17, 2439-2446.

10. Fang, Y.; Chen, V. W.; Cai, Y.; Berrigan, J. D.; Marder, S. R.; Perry, J. W.; Sandhage, K. H. Biologically Enabled Syntheses of Freestanding Metallic Structures Possessing Subwavelength Pore Arrays for Extraordinary (Surface Plasmon-Mediated) Infrared Transmission. Adv. Funct. Mater. 2012, 22, 2550-2559. 
11. Townley, E.; Parker, A. R.; White-Cooper, H. Exploitation of Diatom Frustules for Nanotechnology: Tethering Active Biomolecules. Adv. Funct. Mater. 2008, 18, 369-374.

12. Wang, W.; Gutu, T.; Gale, D. K.; Jiao, J.; Rorrer, G. L.; Chang, C. H. Self-assembly of nanostructured diatom microshells into patterned arrays assisted by polyelectrolyte multilayer deposition and inkjet printing. J. Am. Chem. Soc. 2009, 131, 4178-4179.

13. Zhang, D.; Wang, Y.; Pan, J.; Cai, J. Separation of diatom valves and girdle bands from Coscinodiscus diatomite by settling method. J. Mater. Sci. 2010, 45, 5736-5741.

14. Wang Y, Pan J, Cai J, et al. Floating assembly of diatom Coscinodiscus sp. microshells. Biochemical and biophysical research communications. 2012, 420, 1-5.

15. Osmanlioglu, A. E. Natural diatomite process for removal of radioactivity from liquid waste. Appl. Radiat. Isotopes. 2007, 65, 17-20.

16. Ren, F.; Campbell, J.; Wang, X.; Rorrer, G. L.; Wang, A. X. Enhancing surface plasmon resonances of metallic nanoparticles by diatom biosilica. Optics Express. 2013, 21, 15308.

17. Cottin-Bizonne, C.; Barrat, J. L.; Bocquet, L.; Charlaix, E. Low-friction flows of liquid at nanopatterned interfaces. Nature Materials. 2003, 2, 237-240

18. Maitra, T.; Antonini, C.; Auf der Mauer, M.; Stamatopoulos, C.; Tiwari, M. K.; Poulikakos, D. Hierarchically nanotextured surfaces maintaining superhydrophobicity under severely adverse conditions. Nanoscale. 2014, 7, 8710-8719.

19. Städler, B.; Solak, H. H.; Frerker, S.; Bonroy, K.; Frederix, F.; Vörös, J.; Grandin, H. M. Nanopatterning of gold colloids for label-free biosensing. Nanotechnology. 2007, 18, 155306.

20. Atwater, H. A.; Polman, A. Plasmonics for improved photovoltaic devices. Nat. Mater. 2010, 9, 205-213.

21. Chou, S. Y.; Krauss, P. R.; Renstrom, P. J. Imprint Lithography with 25-Nanometer Resolution. Science. 1996, 272, 85-87.

22. Gates, B. D.; Xu, Q.; Stewart, M.; Ryan, D.; Willson, C. G.; Whitesides, G. M. New approaches to nanofabrication: molding, printing, and other techniques. Chem. Rev. 2005, 105, 1171-1196.

23. Killops, K. L.; Gupta, N.; Dimitriou, M. D.; Lynd, N. A.; Jung, H.; Tran, H.; Bang, J.; Campos, L. M. Strongly Phase-Segregating Block Copolymers with Sub-20 nm Features. ACS Macro Letters, 2013, 2, 677-682.

24. Kim, J. Y.; Kim, B. H.; Hwang, J. O.; Jeong, S. J.; Shin, D. O.; Mun, J. H.; Choi, Y. J.; Jin, H. M.; Kim, S. O. Flexible and transferrable self-assembled nanopatterning on chemically modified graphene. Adv. Mater. 2013, 25, 1331-1335. 